\title{
Beyond toxicity: aryl hydrocarbon receptor-mediated functions in the immune system
}

\author{
Brigitta Stockinger
}

Address: Division of Molecular Immunology, MRC National Institute for Medical Research, Mill Hill, London NW7 1AA, UK. Email: bstocki@nimr.mrc.ac.uk

\begin{abstract}
The aryl hydrocarbon receptor is a ligand-activated transcriptional regulator that binds dioxin and other exogenous contaminants and is responsible for their toxic effects, including immunosuppression. New evidence suggests, however, that the aryl hydrocarbon receptor has a physiological role in the immune system, and the immunosuppressive effects of dioxin may reflect a more subtle disruption of the regulatory interactions between immune cells.
\end{abstract}

The aryl hydrocarbon receptor (AhR), also called the dioxin receptor, is a transcriptional regulator best known for mediating the toxicity of environmental contaminants, most notably halogenated polycyclic aromatic hydrocarbons such as dioxin. AhR has been studied extensively for its pathological role in response to environmental pollution, and there is a wealth of knowledge regarding its signalling components as well as its structural features and pharmacological effects. Although many aspects of AhRmediated toxicity have been described, the molecular mechanisms underlying these are not well understood.

AhR is conserved across vertebrate and invertebrate species, playing a role, for instance, in the development of the nervous system in Caenorhabditis elegans, while in Drosophila the AhR homolog spineless is involved in development of antennae and legs as well as in aspects of color vision [1].The intrinsic physiological functions of AhR in mammals have been delineated from the phenotype of the AhR knockout mouse [2-4]. These mice show reduced fertility, smaller livers, possibly resulting from vascular defects [1], and portal fibrosis. The strong conservation of AhR in so many species as well as the mutant phenotype suggest that it has roles beyond those of mediating toxicity of pollutants. More recently it has been suggested that dioxin-mediated toxicity may, in fact, reflect disruption of the endogenous function of this receptor by inducing sustained and inappropriate AhR signaling due to the stability of the toxin, and thus causing dysregulation of physiological functions [5].

Toxic effects of dysregulation are particularly likely in the immune system where highly complex interactions between hematopoietic cells and their environment dictate the outcome of challenge by pathogens, and indeed dioxin has been known for decades to be immunotoxic, though information on possible underlying mechanisms is sparse [6]. Indications of immune defects have been described in one of the three AhR knockout strains, but not the others, prompting suggestions that differences in background or infectious agents in the environment might have played a role. However, immune challenges such as influenza or Listeria monocytogenes applied to AhR knockout mice have so far yielded little insight into the mechanism of changes that have been reported in the responses of specific subsets of immune cells, or the induction of specific subsets of immune cells [7]. More recent experiments on the expression of $\mathrm{AhR}$ in the lymphocytes of the immune system have begun to suggest that ligands of AhR have roles in the immune system that do not conform to the notion of immunosuppression.

The lymphocytes of the adaptive immune system fall into two major classes - B cells, which secrete antibodies, and $\mathrm{T}$ cells, which act on other cells and, broadly speaking, either activate other cells of the immune system (cells that do this belong to a class known as CD4 $\mathrm{T}$ cells, or $\mathrm{T}$ helper cells) or destroy infected cells (most cells that do this belong to a class known as CD8 T cells). CD4 T cells are further subdivided into four clearly defined subsets with distinct functions that are mediated by the distinct cytokines they secrete and that act on other immune cells (Figure 1), including B cells, which they activate to secrete antibody. All CD4 $\mathrm{T}$ cells to some extent regulate one another's activation, but regulatory $\mathrm{T}$ cells ( $\mathrm{T}_{\mathrm{REG}}$ cells) are specialized for suppressing the other subsets and are thought to be essential for preventing autoimmunity. Clearly, disruption of these regulatory interactions is likely to have complex effects.

It has been assumed on the basis of global microarray analysis of unseparated hematopoietic cell populations that AhR expression is virtually ubiquitous in the immune system [8]. We recently showed, however, that AhR is differentially expressed in different lymphocyte subsets. For instance, in the CD4 $\mathrm{T}$ cell lineage AhR expression is 


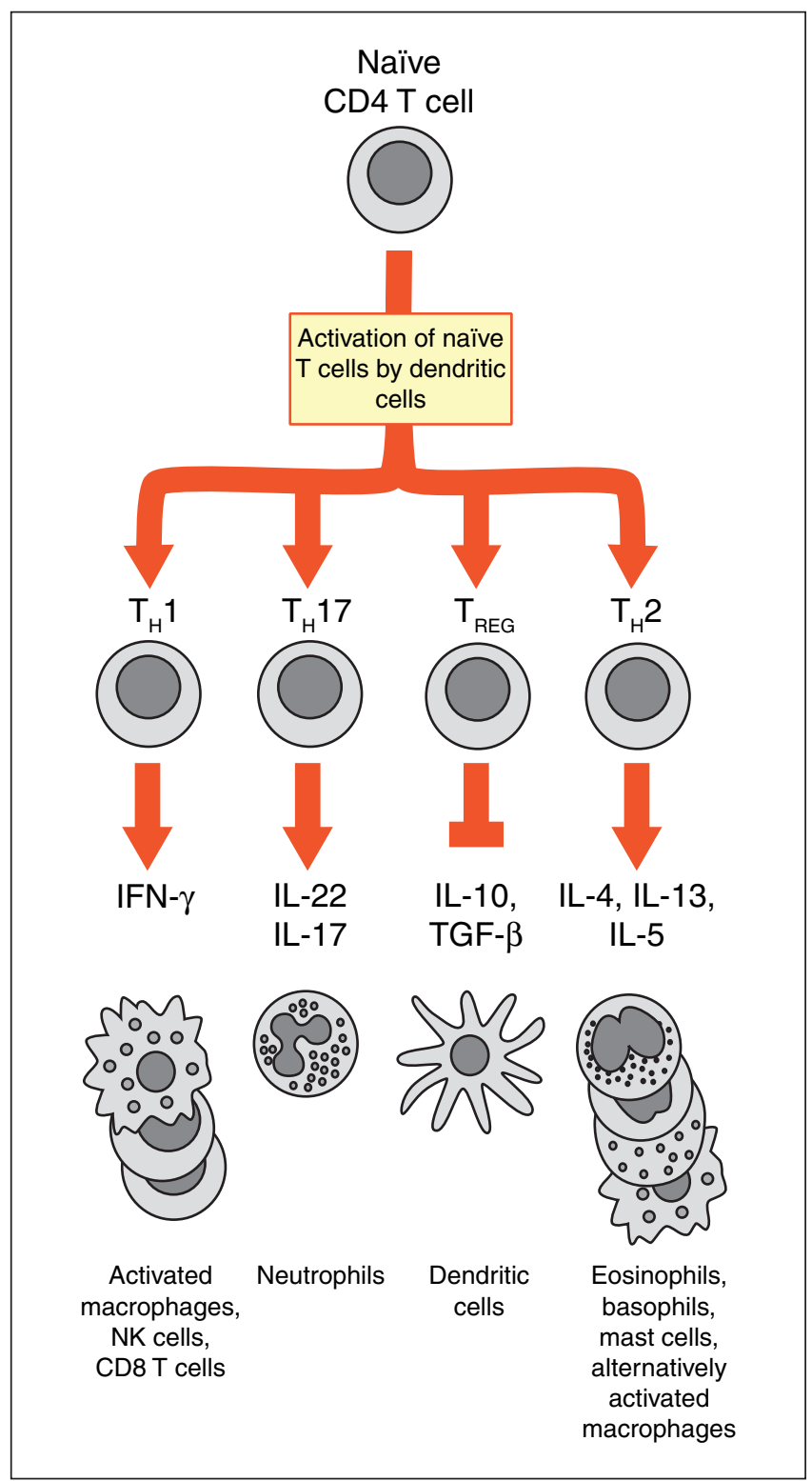

Figure 1

Functional subsets of CD4 T cells. Naïve CD4 T cells - that is, $T$ cells that have not yet been activated by antigen - circulate in the blood and lymph until they are activated, usually by dendritic cells, which are specialized for that function. They then proliferate and differentiate into different functional subsets, distinguished by the different cytokines they produce (indicated under each CD4 T cell type): the cytokines act on other immune cells, activating them in turn. The four known subsets of CD4 T cells are $T_{H} 1$ cells, which induce inflammatory responses that protect the tissues; $\mathrm{T}_{\mathrm{H}} 2$ cells, which are largely responsible for protecting the epithelial surfaces of the gut, lung and genitourinary system; $T_{H} 17$ cells, which produce early inflammatory responses; and $\mathrm{T}_{\mathrm{REG}}$ cells, which inhibit the responses of the other cell types and are thought to provide protection from autoimmune disease. IFN, interferon; IL, interleukin, NK, natural killer; TGF, transforming growth factor. Modified from Figure 5-22 in DeFranco AL, Locksley RM, Robertson M: Immunity: The Immune Response in Infectious and Inflammatory Disease. London: New Science Press; 2007. restricted to $T_{H} 17$ cells [9], whereas it is absent from $T_{H} 1$ and $\mathrm{T}_{\mathrm{H}} 2$ cells and only marginally present in $\mathrm{T}_{\mathrm{REG}}$ cells. AhR expression also varies in other immune cells, including antigen-presenting cells such as dendritic cells and macrophages, which are essential for the activation and some of the effector functions of $\mathrm{T}$ cells (Figure 1), although there is currently little information regarding subset-specific expression.

Thus, the evaluation of microarray analysis or even quantitative PCR of heterogenous cell populations can lead to erroneous conclusions. For instance, expression of AhR in unseparated total $\mathrm{CD} 4 \mathrm{~T}$ cells, which are composed of naïve cells, memory cells, $\mathrm{T}_{\mathrm{H}} 1, \mathrm{~T}_{\mathrm{H}} 2, \mathrm{~T}_{\mathrm{H}} 17$ and $\mathrm{T}_{\mathrm{REG}}$ cells will be detectable at a very low level, and this has prompted the conclusion that AhR is ubiquitously expressed in all CD4 $\mathrm{T}$ cells when, in reality, the signal is caused by high expression in the small subset of $\mathrm{T}_{\mathrm{H}} 17$ cells. Moreover, although expression of $\mathrm{AhR}$ in $\mathrm{T}_{\mathrm{REG}}$ cells is detectable when these cells are assayed on their own [10], by comparison with expression in $\mathrm{T}_{\mathrm{H}} 17$ cells or hepatocytes, this level of expression seems minimal [9], calling into question its physiological relevance.

The differential expression of AhR in immune cells has implications for the physiological functions of this transcriptional regulator. We now know that AhR plays a role in promoting (though not in initiating) the differentiation of $\mathrm{T}_{\mathrm{H}} 17$ cells, and more importantly, in inducing them to secrete the cytokine interleukin (IL)-22 (a cytokine implicated in the defense of mucosal barriers).

AhR-dependent induction of IL-22 is seen with several distinct ligands, so it is not likely that a specific breakdown product rather than the AhR trigger itself is responsible for the effect. Assuming that this reflects a physiological function of AhR, it is not easy to reconcile with the notion that the role of $\mathrm{AhR}$ is to downregulate or suppress immune responses [6]. To resolve this conflict, it has been suggested that the immunosuppressive effects of aryl hydrocarbons are due to the action of $\mathrm{T}_{\mathrm{REG}}$ cells, and that some AhR ligands - for example, dioxin - induce $\mathrm{T}_{\mathrm{REG}}$ cells, whereas others induce $\mathrm{T}_{\mathrm{H}} 17$ cells [10]. I would argue that there are alternative explanations for these findings. There is a tendency in immunology to equate proportional cell representation (given as percentage values) with absolute numbers, and the contention that dioxin induces $\mathrm{T}_{\mathrm{REG}}$ cells is based on this. It seems equally likely, however, that dioxin kills cells - such as $\mathrm{T}_{\mathrm{H}} 17$ cells, as well as other immune cells that may express the receptor at high levels - whereas other AhR ligands do not. In that case, $\mathrm{T}_{\mathrm{REG}}$ cells will be proportionally overrepresented in the CD4 T cell population when exposed to dioxin because, as a consequence of minimal receptor expression, they are likely to escape death by overstimulation. This, however, is far removed from induction, which would mean an 
increase in absolute numbers (rather than percentage) of $\mathrm{T}_{\mathrm{REG}}$ cells. Thus, it is not yet clear that different AhR ligands actually induce different $\mathrm{T}$ cell types.

This issue is of some practical importance because induction of $\mathrm{T}_{\mathrm{REG}}$ cells is a significant target of attempts to suppress autoimmune diseases. For example, it has been suggested that dioxin will suppress experimental allergic encephalitis, a widely used model for multiple sclerosis, through induction of $\mathrm{T}_{\mathrm{REG}}$ cells, a suggestion that is already resulting in a drive to test $\mathrm{AhR}$ ligands for their suppressive effect in this autoimmune model with a view to future application in treatment of the disease.

While the role of AhR in the induction of the cytokine IL-22 is now defined in mice as well as humans, this is just the beginning. We still know little about the physiological impact of AhR expression on other cells of the immune system and the consequences of exposure to AhR ligands for these. Given the complexity of cellular interactions that underlie specific immune responses, the global immune suppression diagnosed after dioxin exposure is likely to hide a complicated network of physiological dysregulation. While dioxin may be the ligand of choice for toxicological studies because of its potency and slow degradation, which reduces the likelihood of 'off-target' effects, I would argue that offtarget effects may well be an integral feature of a tightly orchestrated immune response. Immune responses function in a tight interactive network of cells and mediators, and the behavior of dioxin in the immune system may not reflect direct action on all the cell types that are thought to be affected, and may indeed mask endogenous functions of AhR that are prohibited or perturbed by dioxin. There are multiple physiological ligands for AhR and it is likely that these bind with varying affinities and are rapidly degraded by induced metabolizing enzymes, thus providing transient and tightly regulated stimuli. Amongst these ligands are dietary components - flavones and indoles, tryptophan metabolites as well as intrinsic factors such as prostaglandin subtypes, lipoxin A4, bilirubin and others [11]. It remains to be seen how such endogenous AhR ligands influence various components of the immune system. Unraveling the complex functions of AhR in the immune system, which may also give clues towards crosstalk between the immune system, the neuroendocrine system and the nervous system is more likely to come from models that use physiological ligands whose mode of action is under tight regulatory control.

\section{Acknowledgements}

I would like to thank Dr Alexandre Potocnik for critical comments on this article.

\section{References}

1 McMillan BJ, Bradfield CA: The aryl hydrocarbon receptor sans xenobiotics: endogenous function in genetic model systems. Mol Pharmacol 2007, 72:487-498.

2 Schmidt JV, Su GH, Reddy JK, Simon MC, Bradfield CA: Characterization of a murine Ahr null allele: involvement of the Ah receptor in hepatic growth and development. Proc Natl Acad Sci USA 1996, 93:6731-6736.

3 Fernandez-Salguero PM, Ward JM, Sundberg JP, Gonzalez FJ: Lesions of aryl-hydrocarbon receptor-deficient mice. Vet Pathol 1997, 34:605-614.

4 Mimura J, Yamashita K, Nakamura K, Morita M, Takagi TN, Nakao K, Ema M, Sogawa K, Yasuda M, Katsuki M, FujiiKuriyama Y: Loss of teratogenic response to 2,3,7,8-tetrachlorodibenzo-p-dioxin (TCDD) in mice lacking the Ah (dioxin) receptor. Genes Cells 1997, 2:645-654.

5 Bock KW, Kohle C: Ah receptor: dioxin-mediated toxic responses as hints to deregulated physiologic functions. Biochem Pharmacol 2006, 72:393-404.

6 Kerkvliet NI: AHR-mediated immunomodulation: the role of altered gene transcription. Biochem Pharmacol 2009, 77: 746-760.

7 Esser C: The immune phenotype of AhR null mouse mutants: not a simple mirror of xenobiotic receptor overactivation. Biochem Pharmacol 2009, 77:597-607.

8 Frericks M, Meissner M, Esser C: Microarray analysis of the AHR system: tissue-specific flexibility in signal and target genes. Toxicol Appl Pharmacol 2007, 220:320-332.

9 Veldhoen M, Hirota K, Westendorf AM, Buer J, Dumoutier L, Renauld JC, Stockinger B: The aryl hydrocarbon receptor links TH17-cell-mediated autoimmunity to environmental toxins. Nature 2008, 453:106-109.

10 Quintana FJ, Basso AS, Iglesias AH, Korn T, Farez MF, Bettelli E, Caccamo M, Oukka M, Weiner HL: Control of T(reg) and $\mathrm{T}(\mathrm{H}) 17$ cell differentiation by the aryl hydrocarbon receptor. Nature 2008, 453:65-71.

11 Denison MS, Nagy SR: Activation of the aryl hydrocarbon receptor by structurally diverse exogenous and endogenous chemicals. Annu Rev Pharmacol Toxicol 2003, 43:309334.

Published: 17 August 2009

doi:10.1186/jbiol170

(C) 2009 BioMed Central Ltd 